\title{
On the Analysis of Hyper-Parameter Space for a Genetic Programming System with Iterated F-Race
}

\author{
Leonardo Trujillo . \\ Ernesto Álvarez \\ González • Edgar \\ Galván · Juan J. \\ Tapia · Antonin \\ Ponsich
}

Received: date / Accepted: date

\begin{abstract}
Keywords Hyper-Parameter Optimization · Iterated F- Race · Genetic Programming

Evolutionary Algorithms (EAs) have been with us for several decades and are highly popular given that they have proven competitive in the face of challenging problems' features such as deceptiveness, multiple local optima among other characteristics. However, one needs to define multiple hyper-paramater values to have a useful EA, this is a drawback for many practitioners. In particular, only until recently has hyperparameter optimization been extensively studied for genetic programming (GP), an EA for the evolution of models and programs. This work builds on recent findings, and explores the hyper-parameter space of a specific GP system called neat-GP that controls model
\end{abstract}

Leonardo Trujillo · Ernesto Álvarez González

Tecnológico Nacional de México/I.T. Tijuana, Tijuana, BC, Mexico

E-mail: leonardo.trujillo@tectijuana.edu.mx

E-mail: emmalv365@gmail.com

Edgar Galván

National University of Ireland, Maynooth, Ireland

E-mail: edgar.galvan@mu.ie

Juan J. Tapia

Instituto Politécnico Nacional - CITEDI, Av. Instituto Politécnico Nacional No. 1310 Colonia Nueva Tijuana, C.P. 22435, Tijuana, B.C., Mexico

E-mail: jjtapia@citedi.mx

\section{Antonin Ponsich}

UAM (Azcapotzalco) - Universidad Autónoma Metropolitana, Av. San Pablo No. 180, Col. Reynosa Tamaulipas, C.P. 02200, CDMX, Mexico

E-mail: aspo@correo.azc.uam.mx size (bloat). This is done using two large sets of symbolic regression benchmarks to evaluate system performance, while hyper-parameter optimization is carried out using three variants of the iterated F-Race algorithm, for the first time applied to GP. From all the automatic parametrizations found, several findings are notable. Automatic parametrizations do not outperform the manual configuration in any substantial way based on testing error. Moreover, finding parametrizations that produce highly accurate models that are also compact is not trivially done, at least if the hyperparameter optimization process (F-Race) is only guided by predictive error. This work is intended to foster more research and scrutiny of hyper-parameters in EA in general and GP in particular.

\section{Introduction}

Hyper-parameters are configuration variables that regulate or control the behavior of an optimization or learning algorithm (Birattari, 2009; Sipper et al, 2018). For evolutionary algorithms (EAs), hyperparameters include population size, mating rates, number of generations, and other 1 . In all metaheuristic search techniques, the proper setting of hyperparameter values can be a difficult and tedious endeavor (Birattari, 2009). This is also true for EAs that often lack a theoretical foundation to derive their optimal parametrization values analytically, with few exceptions (Hansen and Ostermeier, 2001). This work focuses on hyper-parameter tuning or optimization (Birattari, 2009; Neumüller et al, 2012), also referred to as meta-optimization, which is performed offline, in contrast with hyper-parameter control where an EA or any other meta-heuristic algorithm dynamically modifies the hyper-parameter values during online execution (Karafotias et al, 2015).

This topic has been studied extensively for EAs, but has received less attention in genetic programming (GP) literature (Koza, 1992; Langdon and Poli, 2010). In GP, an EA is used to search for models, functions or small segments of code that perform a particular computational process, usually following a supervised learning problem formulation. Probably the most extensive experimental study regarding hyper-parameter optimization for GP was recently published by Sipper et al (2018). The authors also present an up to date survey on this topic.

\footnotetext{
1 They are also referred to as parameters, but the distinction between parameters and hyper-parameters is important, particularly when the EA is performing a learning process, searching for models that might also include their own parameters.
} 
Sipper et al (2018) evaluate two different GP systems using several standard GP benchmarks and supervised learning (classification) benchmarks. Two hyperparameter optimization procedures are tested, an EA (meta-evolution) and random search. They consider common hyper-parameters for EAs and GP, such as: population size, number of generations, crossover rate, mutation rate and tournament size. The authors performed an extensive number of GP runs to gather their results, allowing them to draw interesting insights and suggestions. Surprisingly, random search seems to be as good as an EA for this task, and at least two findings reported by the authors are noteworthy and very useful for practitioners and applied researchers. First, it seems that good parametrizations (specific combination of hyper-parameter values) can be found all over hyper-parameter space; i.e. good parameter settings can be quite diverse, they are not clustered in specific regions of hyper-parameter space. Second, while finding general hyper-parameter values (that are applicable to several different problems) is more difficult compared to finding good parameter values for a single problem instance. This can be done efficiently through random search.

On the other hand, there are some aspects that were not considered by Sipper et al (2018). First, the authors do not account for the fact that most problems that are solved with GP are supervised learning problems. In such cases, fitness is basically proportional to training performance, which is the measure used to evaluate different hyper-parameter configurations. However, of more interest to practitioners is performance on an unseen test set of fitness cases, this, however, is not considered in (Sipper et al, 2018). Second, the study focuses on very general hyper-parameters, it does not consider system-specific parameters, even for the more sophisticated GP system studied (Cava et al, 2019). Third, the work does not explore the effect that different parametrizations have on solution size, an important aspect in most GP-based systems (Trujillo et al, 2016). Finally, there are other state-of-the-art methods for hyper-parameter optimization, namely racing algorithms (Birattari et al, 2002; Lpez-Ibez et al, 2016), that can be used for this task besides a basic EA and random search.

Therefore, the present paper is intended as a complimentary study to the research reported by Sipper et al (2018), with the following main contributions.

- Hyper-parameter space is explored for a bloat-free GP system called neat-GP (Trujillo et al, 2016). Two common EA hyper-parameters are considered (crossover rate and mutation rate), as well as three system-specific hyper-parameters of neat-GP.
- Three variants of the iterated F-Race algorithm are evaluated, using two extensive sets of 15 easy and 16 hard benchmark problems.

- Results are evaluated considering testing performance, solution size and best parameter values found, all relative to the manual configuration suggested in (Truijllo et al, 2016).

- Results are contrasted with those reported in (Sipper et al, 2018), showing agreement in some results while diverging in other relevant aspects.

The remainder of this paper is organized as follows. Section 2 provides a brief overview of required background, discussing the general principles of hyperparameter optimization and the basics of racing algorithms. Section 3 presents a summary of the neat-GP system, and discusses the hyper-parameters considered in this study. Afterward, Section 4 presents the experimental work and detailed results. Section 5 contains a discussion of the results, and concluding comments are in Section 6 .

\section{Background}

The goal in the section is to provide a brief introduction to the problem of hyper-parameter optimization and to the racing algorithms used in this work, namely several variants of the iterated F-Race. However, for a more thorough account of these subjects the interested reader should see (Birattari, 2009; Neumüller et al, 2012; Sipper et al, 2018) for the former, and (Birattari et al, 2002; Lpez-Ibez et al, 2016) for the latter.

\subsection{Hyper-Parameter Optimization}

The process of hyper-parameter optimization is also known as meta-optimization, automatic parameter tuning and, in the case of an EA, meta-evolution. It consists in using an optimization algorithm to automatically tune the hyper-parameters of another algorithm, such that the expected performance on a given (set of ) problem(s) can be maximized (or minimized as the case may be). In other words, an optimization algorithm, which can be referred to as the metaoptimizer $M O$, searches for an optimal set (or vector) of hyper-parameter values $\theta$ that represent a particular parametrization for an optimization or learning algorithm $A$, such that $A$ achieves the best possible performance on a set of problems $\left\{P_{i}\right\}$, with $i=1, \ldots, n$.

To perform hyper-parameter optimization, the following must be defined: 
1. The algorithm $A$ that will be tuned.

2. The hyper-parameters of $A$ that are considered in $\theta$.

3. The meta-optimizer $M O$ to be used.

4. The set(s) of problems $\left\{P_{i}\right\}$ that are used to evaluate the quality of different parametrizations $\theta$; these can be thought off as meta fitness cases in a GP sense.

Some comments regarding how previous works relate to the three points mentioned above are relevant. Hyper-parameter optimization is now widely known, but often not used due to the associated computational cost. It has been applied to a wide variety of optimization algorithms, meta-heuristic search techniques and machine learning algorithms. However, one class of algorithms has received relatively much less attention, namely GP. Probably the most notable study regarding GP is (Sipper et al, 2018), as discussed above.

Regarding the hyper-parameters that are optimized, these can be numerical, categorical or ordinal parameters. In the case of an EA or GP, numerical parameters can be population size or number of total generations, categorical parameters can refer to the type of crossover that is used, while ordinal parameters are similar to categorical but with an implicit ordering. This work focuses on numerical parameters, following (Sipper et al, 2018).

With regards to the type of meta-optimizer, there is a variety of methods in current literature, most of which are gradient-free heuristics or meta-heuristics, experimental design techniques or statistical modeling approaches. Probably the simplest approach is to perform a random search (Bergstra and Bengio, 2012) or a grid search (Olson et al, 2017), which are easy to implement, tend to achieve good performance improvements compared to manual tuning, and are widely used. Another common strategy is to use EAs, as in (Sipper et al, 2018). However, one class of algorithms that has proven to be particularly successful are racing approaches (Birattari et al, 2002; Lpez-Ibez et al, 2016), since they deal with one of the main issues in hyper-parameter optimization, which is the computational cost. Performing hyper-parameter optimization is often avoided because it requires multiple executions (using different parameter values) of the algorithm to be tuned. Moreover, meta-heuristics are often stochastic search techniques, meaning that their performance level for solving one problem instance has to be evaluated in terms of some statistical measure (for instance, mean or median value) computed over several executions of the algorithm. This increases the computational burden of performing hyper-parameter optimization. This is particularly problematic for EAs in general, and GP in particular, which are notoriously plagued by long run times. Racing algorithms perform a race between different candidate parametrizations, and discard those parametrizations that show the weakest performance over a subset of the problems used for evaluation; these methods are further discussed in the following subsection.

Finally, racing algorithms, and more precisely iterated versions of the algorithm, have not been previously used to tune a GP system. This paper presents the first extensive study that considers this combination, using a diverse set of widely accepted benchmarks for symbolic regression with GP (McDermott et al, 2012).

\subsection{F-Race and Iterated F-Race}

In racing algorithms for hyper-parameter optimization, a set of candidate parametrizations $\left\{\theta_{j}\right\}$, with $j=1, \ldots, m$, are generated using a given distribution, with $\theta_{j}=\left[x_{1}, \ldots x_{p}\right], x_{u}$ the $u$-th hyper-parameter, and $p$ the total number of hyper-parameters. For instance, if we define $X$ as the space of valid parametrizations considered in a given study, then $\left\{\theta_{j}\right\}$ can be constructed by uniform random sampling of $X$, using an independent distribution for each hyper-parameter. Then, each parametrization is sequentially evaluated on each problem from set $\left\{P_{i}\right\}$. For each parametrization $\theta_{j}$, a performance or cost vector is constructed of the form $C\left(\theta_{j},\left\{P_{i}\right\}\right)=\left[C_{j}\left(P_{1}\right), C_{j}\left(P_{2}\right), \ldots\right]$, where $C_{j}\left(P_{i}\right)$ represents the performance of parametrization $\theta_{j}$ applied to problem $P_{i}$. After a certain number of problems $1 \leq h \leq n$ have been evaluated, and after each subsequently tested problem, the parametrizations are compared according to their accumulated performance vector $\left[C_{j}\left(P_{1}\right), \ldots, C_{j}\left(P_{h}\right)\right]$. Depending on the racing algorithm used, a specific criterion is used to eliminate the worst performing parametrizations based on their accumulated performance vectors. In the case of F-Race (Birattari et al, 2002; Lpez-Ibez et al, 2016), the criterion for removal from the race is based on Friedmans non-parametric two-way analysis of variance by ranks. In particular, if a parametrization $\theta_{j}$ is performing statistically worse than at least one other parametrization in the race then $\theta_{j}$ is deleted. In the case of F-Race, $h$ is set to 3 such that the Friedmans test has sufficient evidence within the corresponding performance vectors to determine if a statistically significant difference exists between parametrizations. At the end of the run, F-Race returns the subset $\Theta^{O} \subset\left\{\theta_{j}\right\}$ of parametrizations that contains all of the statistically superior and equivalent parametrizations, also referred to as elite parametrizations. 
A common extension to the F-Race algorithm consists in performing the racing process multiple times sequentially, what is referred to as the iterated F-Race or iF-Race (Lpez-Ibez et al, 2016). The algorithm returns the set $\left\{\bigcup \Theta_{l}^{O}\right\}$ with $l=1, \ldots, s$ and $\Theta_{l}^{O}$ being the optimal set of parametrizations returned after iteration $l$, with the process executed a total of $s$ iterations. The iF-Race algorithm is a direct extension of the FRace algorithm, where the crucial step is in how the set $\left\{\theta_{j}\right\}$ is generated at the beginning of each iteration. In particular, three variants are considered in the present paper, following (Lpez-Ibez et al, 2016).

1. Original iF-Race. This variant creates a new set $\left\{\theta_{j}\right\}$ for each iteration of iF-Race using a uniform distribution over $X$, executing several independent F-Race executions. A useful feature is that each F-Race execution can be run in parallel. However, there is an obvious loss of information between runs that might be useful to make the racing process more efficient. This observation justifies the strategies in the following two variants.

2. iF-Race with Elitism. In this case, the set $\Theta_{l}^{O}$ is used to seed the set $\left\{\theta_{j}\right\}$ for iteration $l+1$. If $\left|\Theta_{l}^{O}\right|<$ $m$, then the remaining $m-\left|\Theta_{l}^{O}\right|$ parametrizations are randomly generated using a uniform distribution like in the original iF-Race.

3. iF-Race with Gaussian update. This strategy is based on (Lpez-Ibez et al, 2016), with minor changes to simplify the process. As with iF-Race with Elitism, the set $\Theta_{l}^{O}$ is used to seed the set $\left\{\theta_{j}\right\}$ for iteration $l+1$. However, new parametrizations are generated as perturbations from the parametrizations returned in $\Theta_{l}^{O}$. First, with equal probability for each elite configuration, a single elite parametrization $\theta_{e}$ is chosen from $\Theta_{l}^{O}$. A new parametrization is generated by sampling a normal distribution for each hyper-parameter $x_{u}$, using the value of $x_{u}$ in $\theta_{e}$ as the mean and setting the standard deviation $\sigma_{u}^{l}=\left(x_{\max }-x_{\min }\right) / 2$, and decreasing the value for each subsequent iteration $l$ by $\sigma_{u}^{l}=\sigma_{u}^{l-1}\left(m-\left|\Theta_{l-1}^{O}\right|\right)^{(-1 / p)}$ with $p$ the total number of hyper-parameters to be tuned.

In summary, three variants of iF-Race are used in this work, which we respectively refer to as Original, Elitism and Gaussian for simplicity. If we consider iFRace as a search process, then the methods range from a more explorative process (Original), to a more greedy exploitative approach (Gaussian). In the first one, the process repeats the F-Race algorithm for $s$ iterations. The Elitism strategy is a more greedy approach since the set of parametrizations used by F-Race at each iteration is seeded by the best parametrizations found in previous iterations. This allows the algorithm to be more efficient, by quickly discarding bad parametrizations as the iF-Race process progresses. Finally, the Gaussian approach increases the level of exploitation in the search, by not only using the best parametrizations from previous iterations, but also by generating new parametrizations as tweaked versions of these elite parametrizations, similar to what is done in a random walk with Gaussian perturbation.

\section{3 neat-GP: Bloat Free Genetic Programming}

In general, neat-GP is a bloat-control GP system that is based on the neuroevolution of augmenting topologies algorithm (NEAT) (Stanlev and Miikkulainen, 2002), achieving strong results in regression, classification and computer vision problems (Trujillo et al, 2016; Hernandez-Beltran et al, 2019). A traditional GP system uses a variable-size tree-based representation for candidate solutions, which are syntax trees that encode a particular model, function or operators, or more generally an individual program that is a solution candidate. Trees are composed by elements from a finite set of terminal elements (terminal set) that can be used as leaves in individual trees and that represent problem inputs, while internal nodes contain elements from a finite set of functions (function set) that represent basic operators that can be used by the GP system to construct individual models. Bloat is the tendency of GP to generate unnecessarily large solutions (large syntax trees), even when larger solutions do not provide a proportional improvement in fitness. In general, bloat is seen as a problem that needs to be overcome in most GP systems, since it produces solutions that are more complex and difficult to interpret, while also slowing down the search process since larger solutions require more computational resources to be evaluated.

The main features of neat-GP are the following: The initial population only contains shallow GP trees (3 levels, with the root at level 1), while most GP algorithms initialize the search with small and medium sized trees (depth between 3 and 6 levels). The NEAT approach is to start with simple (small) solutions and to progressively build complexity (add size) as the search progresses. The population is divided into species, such that each species contains individuals of similar size and shape; this process is called speciation, which protects innovation during the search. The algorithm uses fitness sharing, whereby the fitness of individuals is penalized proportionally to the size of the species to which it belongs. This allows the search to maintain an heterogeneous population of individuals based on their size. The only exceptions are the best individuals from each 
species, these are not penalized to allow the search to maintain the best candidate solutions for the problem. This is the last element of neat-GP, a strong selection pressure that uses elitism frequently.

\subsection{Speciation, Tree Dissimilarity and Fitness Sharing}

In neat-GP, individuals are grouped together into species based on their size and shape. For a tree $T$ let $n_{T}$ represent the size of the tree (number of nodes) and $d_{T}$ represents its depth (number of levels). Moreover, let $S_{i, j}$ represent the shared structure between two trees $T_{i}$ and $T_{j}$, starting from the root node (upper region of the trees), which is also a tree. Then, the dissimilarity between two trees $T_{i}$ and $T_{j}$ is given by

$\delta_{T}\left(T_{i}, T_{j}\right)=\beta \frac{N_{i, j}-2 n_{s_{i, j}}}{N_{i, j}-2}+(1-\beta) \frac{D_{i, j}-2 d_{s_{i, j}}}{D_{i, j}-2}$

where $N_{i, j}=n_{T_{i}}+n_{T_{j}}, D_{i, j}=d_{T_{i}}+d_{T_{j}}$, and $\beta \in[0,1]$.

Using the above measure, individuals are grouped into species based on their size and depth. When a new individual $T_{i}$ is generated, it is compared with a single random individual $T_{j}$ from each species in sequence. Starting with a random ordering of the species, if $\delta_{T}\left(T_{i}, T_{j}\right)<h$, with threshold $h$ being an algorithm parameter, then $T_{i}$ is assigned to the same species as $T_{j}$ and no more comparisons are done. If the previous conditions are never met, then a new species is created for $T_{i}$. Fitness sharing is used in each species, such that individuals that belong to large species are penalized while individuals that belong to smaller ones are less so.

The best individual of each species will be an exception, the fitness of this solution is not penalized to preserve elite solutions within the population. The penalization only becomes important in the parent selection step, which is done deterministically by sorting the population according to the adjusted fitness, making the process more elitist compared to traditional GP. Thus, highly penalized individuals might not produce any offspring. However, if the best solution in a species is good enough, it can still survive and produce offspring since it is not affected by the penalization process.

\subsection{Selection, genetic operators and survival}

Parent selection uses an elitist strategy, such that the $p_{\text {worst }} \%$ individuals of each species are deleted, and the same number of offspring are generated to replace them. A maximum number of possible offspring for each individual is set proportionally to its raw fitness. Afterward, individuals are ranked based on their adjusted fitness and selected in order, randomly choosing whether to generate offspring by way of crossover or mutation. Each time an individual is selected as a parent, its expected number of descendants is reduced accordingly, and when this value reaches zero the individual cannot be used again as a parent.

\subsection{Hyper-Parameter Optimization for neat-GP}

In the present study, we choose to focus on the following hyper-parameters of neat-GP:

1. Crossover rate $\left(C_{p}\right)$ and mutation rate $\left(M_{p}\right)$. Since new individuals are only created through these two genetic operators, and since the operators are mutually exclusive in neat-GP, then both hyperparameters can be defined with one numerical value given that $M_{p}=1-C_{p}$.

2. Species criteria $\beta$. This is the short name for the $\beta$ hyper-parameter in Equation 1, which defines the relative importance in the similarity measure used to consider both the size and depth of trees when they are compared in the speciation process.

3. Species threshold $h$. This hyper-parameter controls the creation of new species during the search and can be thought of as a niche radius in an EA framework.

4. Elitism $p_{\text {worst }} \%$. The hyper-parameter determines the amount of elitism used by the search in each individual species.

Table 1 summarizes the above-mentioned neatGP hyper-parameters, and also shows the manual values defined for these parameters in earlier works (Trujillo et al, 2016; Hernandez-Beltran et al, 2019) in the second column from left to right. Moreover, the third column shows the range of values considered by the iF-Race algorithms during the hyper-parameter optimization reported in the next section. Note that for $h$ and $p_{\text {worst }} \%$, in theory both hyper-parameters could have values between 0 and 1 . However, smaller ranges are used in this study for two reasons. In a series of preliminary tests, when these hyper-parameters took values outside the range specified in Table 1 then the search process produced very poor results or extremely bloated solutions. Second, this allows the iF-Race optimization to focus on more promising regions of Hyperparameter space, making the process more efficient.

\section{Experiments}

The goal of the experimental work is to characterize the hyper-parameter space of the neat-GP algorithm. Three variants of iF-Race are considered, namely the Original 
Table 1: The neat-GP hyper-parameters, showing the manual configuration reported in (Trujillo et al, 2016; Hernandez-Beltran et al, 2019) and the range of values considered by the iF-Race optimization.

\begin{tabular}{lll}
\hline Parameter & Manual Setting & Optimization range \\
\hline Crossover probability $C_{p}$ & 0.7 & {$[0-1]$} \\
Mutation probability $M_{p}$ & 0.3 & $1-C_{p}$ \\
Species criteria $\beta$ & 0.5 & {$[0-1]$} \\
Species threshold $h$ & 0.15 & {$[0.05-0.40]$} \\
Elitism $p_{\text {worst }} \%$ & 0.5 & {$[0.2-0.8]$}
\end{tabular}

Table 2: First set of easy benchmark problems: Koza \& Nguyen

\begin{tabular}{llll}
\hline ID & Name & Input Variables & Function \\
\hline P1 & Koza-1 & 1 & $x^{4}+x^{3}+x^{2}+x$ \\
P2 & Koza-2 & 1 & $x^{5}-2 x^{3}+x$ \\
P3 & Koza-3 & 1 & $x^{6}-2 x^{4}+x^{2}$ \\
P4 & Nguyen-1 & 1 & $x^{3}+x^{2}+x$ \\
P5 & Nguyen-2 & 1 & $x^{4}+x^{3}+x^{2}+x+1$ \\
P6 & Nguyen-3 & 1 & $x^{5}+x^{4}+x^{3}+x^{2}+x$ \\
P7 & Nguyen-4 & 1 & $x^{6}+x^{5}+x^{4}+x^{3}+x^{2}+x$ \\
P8 & Nguyen-5 & 1 & $\sin \left(x^{2}\right) \cos (x)-1$ \\
P9 & Nguyen-6 & 1 & $\sin (x)+\sin \left(x+x^{2}\right)$ \\
P10 & Nguyen-7 & 1 & $\ln (x+1)+\ln \left(x^{2}+1\right)$ \\
P11 & Nguyen-8 & 1 & $\sqrt{x}$ \\
P12 & Nguyen-9 & 2 & $\sin (x)+\sin \left(y^{2}\right)$ \\
P13 & Nguyen-10 & 2 & $2 \sin (x)+\sin \left(y^{2}\right)$ \\
P14 & Nguyen-11 & 2 & $x^{y}$ \\
P15 & Nguyen-12 & 2 & $x^{4}-x^{3}+\frac{y^{2}}{2}-y$ \\
& & &
\end{tabular}

formulation, and the Elitism and Gaussian approaches described before. The test problems are described in the following subsection, along with the experimental setup.

\subsection{Problems and Experimental Setup}

Two sets of benchmark problems from (McDermott et al, 2012) are used, shown in Table 2 and Table 3 . All of the problems define symbolic regression tasks, the most common application domain of GP; i.e. learning problems with one or several real-valued inputs and a single real-valued output, defined by a training set used to compute fitness and a test set to validate the performance of the best model found. The first set of problems, which consists of the 15 Koza \& Nguyen problems in Table 2, are considered to be easy problems, while the second set of problems, the 16 Pagie \& Korns problems in Table 3] present more difficult regressions tasks.

For all problems the goal is to optimize the hyperparameter in Table ??, the remaining hyper-parameters were set as follows. In all runs, neat-GP used a population of 100 individuals and 100 generations. Fitness was computed based on the training set of fitness cases using the root mean squared error (RMSE) (thus defining a
Table 3: Second set of hard benchmark problems: Pagie \& Korns

\begin{tabular}{llll}
\hline ID & Name & Input Variables & Function \\
\hline P1 & Pagie-1 & 2 & $\frac{1}{1+x^{4}}+\frac{1}{1+y^{4}}$ \\
P2 & Korns-1 & 2 & $1.57+(24.3 v)$ \\
P3 & Korns-2 & 5 & $0.23+14.2 \frac{v+y}{3 w}$ \\
P4 & Korns-3 & 5 & $-5.41+4.9 \frac{v-x+\frac{y}{w}}{3 w}$ \\
P5 & Korns-4 & 5 & $-2.3+0.13 \sin (z)$ \\
P6 & Korns-5 & 5 & $3+2.13 \ln (w)$ \\
P7 & Korns-6 & 5 & $1.3+0.13 \sqrt{x}$ \\
P8 & Korns-7 & 5 & $213.80940889\left(1-e^{-0.54723748542 x}\right)$ \\
P9 & Korns-8 & 5 & $6.87+11 \sqrt{7.23 x v w}$ \\
P10 & Korns-9 & 5 & $\frac{\sqrt{x}}{v^{2}}$ \\
P11 & Korns-10 & 5 & $0.81+24.3 \frac{2 y+3 z^{2}}{4 v^{3}+5 w^{4}}$ \\
P12 & Korns-11 & 5 & $6.87+11 \cos \left(7.23 x^{3}\right)$ \\
P13 & Korns-12 & 5 & $2-2.1 \cos (9.8 x) \sin (1.3 w)$ \\
P14 & Korns-13 & 5 & $32-3 \frac{\tan (x)}{\tan (y)} \frac{\tan (z)}{\tan (v)}$ \\
P15 & Korns-14 & 5 & $22-4.2(\cos (x)-\tan (y)) \frac{\tanh (z)}{\sin (v)}$ \\
P15 & Korns-15 & 5 & $12-6 \frac{\tan (x)}{e^{y}}(\ln (z)-\tan (v))$
\end{tabular}

minimization problem). The terminals for all GP trees were the input variables of the problems and random ephemeral constants, and the function set was defined as $\{+,-, \times, \sin , \cos , \log$, sqrt, $\tan , \tanh \}$. In all cases, the training and testing sets were sampled following (McDermott et al, 2012).

Given that three variants of iF-Race are considered (Original, Elitism and Guassian), and two sets of problems (Koza \& Nguyen and Pagie \& Korns), six different sets of experiments are carried out. In all cases, training RMSE is used to compare solutions during racing, but testing performance is also recorded and reported below. For all iF-Race variants, a total of $s=10$ iterations are performed, and a set of 200 random parametrizations are used to initialize each race.

The number of total neat-GP runs executed during a single iF-Race experiment is large. Take for instance the most extreme case, applying the Original iF-Race on the 16 Pagie \& Korns problems. For each iteration of the race, 200 candidate parametrizations are considered initially. If at the end of an iteration there are, for example, at least 30 elite parametrizations, then the total number of runs is greater than $30 \times 16 \times 10=4,800$ runs for that single experiment. Given this high computational cost, the entire system was implemented using the DEAP (De Rainville et al, 2012) implementation of neat-GP (Juárez-Smith and Trujillo, 2016) 2, using Python 2.7 and parallelized using Message Passing Interface (MPI) mpi4py Python package running over Ubuntu 16.04, on a cluster with five nodes, where each node had a four core Intel i5-4590 CPUs @3.30 $\mathrm{GHz}$ and $8 \mathrm{~GB}$ RAM. The server node functioned as the administrator in charge of distributing independent

2 https://github.com/saarahy/NGP-LS 
Table 4: Total elite parametrizations produced in each experiment.

\begin{tabular}{lll}
\hline Problem set & iF-Race Variant & Total Parametrizations \\
\hline Koza \& Nguyen & Original & 31 \\
Koza \& Nguyen & Elitism & 61 \\
Koza \& Nguyen & Gaussian & 42 \\
Pagie \& Korns & Original & 612 \\
Pagie \& Korns & Elitism & 227 \\
Pagie \& Korns & Gaussian & 45
\end{tabular}

threads of single neat-GP executions and also collects all the results from the client nodes.

\subsection{Results}

This sections provides a detailed presentation of the main results. Table 4 presents a summary of the total number of elite parametrizations found in each experiment (each iF-Race variant and each problem set). Notice two trends. First, the number of parametrizations produced in the easier set of problems, Koza \& Nguyen, is smaller than on the more difficult set when using the Original and Elitism iF-Race variants. This suggests that there are in fact some elite parametrizations that quickly dominate the racing process for these problems. On the other hand, for the more difficult problems, Pagie \& Korns, it seems to be more difficult to find parametrizations that dominate the F-Race process; i.e. it is more difficult to find parametrizations that are good for all the problems. In the case of iF-Race with Gaussian update, the number of elite paramtrizations is the same for both problem sets. This result, however, may be better explained by the greedy nature of this optimization process, compared to the behavior of the Original and Elitism variants. The more explorative Original iF-Race is able to randomly find many parametrizations that cannot be discarded during the racing process. This makes sense, and is corroborated by some of the results presented in (Sipper et al, 2018), where the authors concluded that for difficult problems it is feasible to find random parametrizations that produce good results, but most of these parametrizations will not generalize to other problems. This highlights the need for hyper-parameter optimization in difficult real-world scenarios.

\subsubsection{Sampling density hyper-parameter space}

Figure 1 and Figure 2 present density plots for each hyper-parameter produced by each experiment. The plots show how the elite parametrizations produced by each iF-Race variant are clustered in hyper-parameter space. For reference, the plots also show the manual setting (as a vertical line), as suggested in the original neat-GP paper (Trujillo et al, 2016). Several trends are noticeable in these plots. First, the Gaussian variant produces narrower density plots, but this is more apparent for two hyper-parameters in particular: crossover probability and species criteria. This applies for both problem groups. Second, the manual configuration shows up very close to the maximum of the density curves for two hyper-parameters: species criteria and elitism percentage. This suggests good agreement between the iF-Race optimization and the manual configuration for these hyper-parameters. On the other hand, crossover probability, and to a lesser extent species threshold show larger deviations between the maxima of the iF-Race density and the manual configuration.

A more detailed view of the elite hyper-parameters found by each iF-Race variant are shown in the radar plots of Figure 3 and Figure 4. The plots highlight all of the elite parametrizations produced by the racing process, and as a darker line the manual configuration for reference.

\subsubsection{Test RMSE and solution size}

It is also relevant to analyze the performance of the elite parametrizations produced by iF-Race, based on performance and size of the models generated by neatGP. Performance is measured by the testing RMSE of the best model found (remember that the racing elimination process was based on training RMSE), while solution size is computed as the average number of nodes of all individuals in the final population of the neat-GP evolution. Figure 5 presents the testing RMSE achieved on each problem in the Koza \& Nguyen set, using the IDs of Table 2, Figure 6] shows the same information for the Pagie \& Korns set of problems. The results are shown as a parallel coordinates plot, where each curve represents one elite parametrization and the vertical axis represent each benchmark problem. In all of these plots, lower is better since the goal is to minimize error.

Notice that while the automatic parametrizations do outperform the manual parametrization on some problems, this is not always the case. In fact, on most problems the performance of the manual configuration and the elite parametrizations produced by iF-Race are more or less equivalent. While this may seem counter intuitive, it is important to remember that this comparison is based on testing RMSE, and that iF-Race is based on training RMSE. Hence, while iF-Race pushes the search for optimal hyper-parameters towards better 


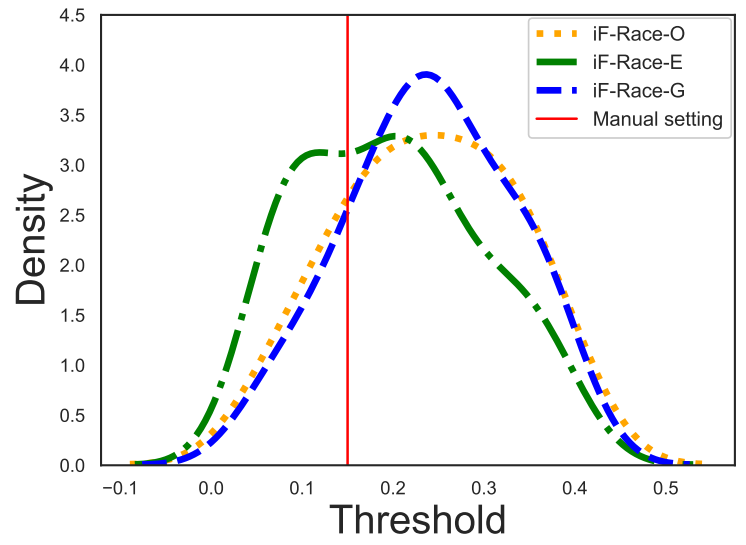

(a)

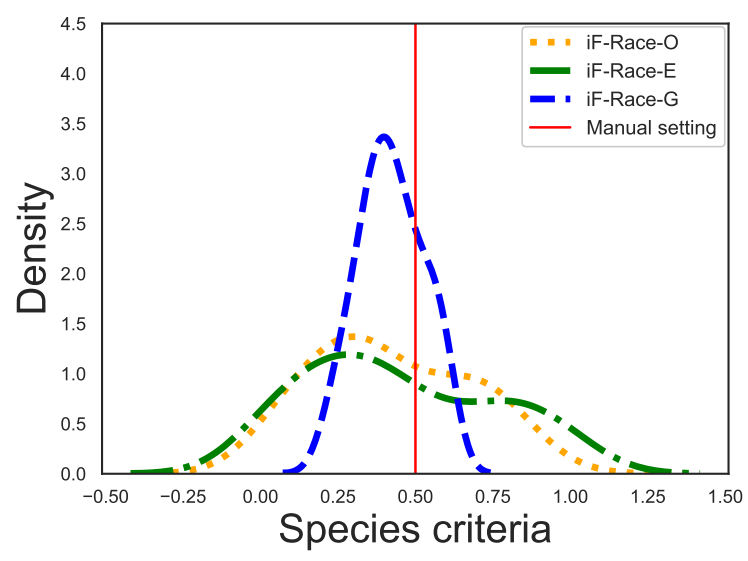

(c)

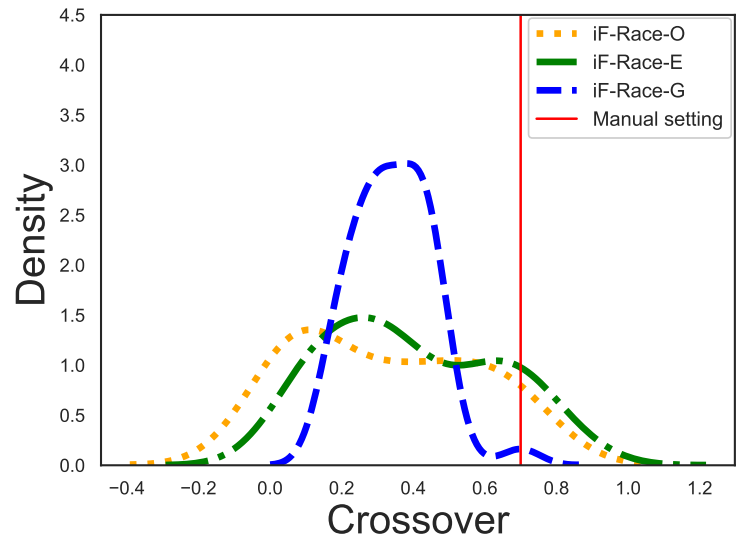

(b)

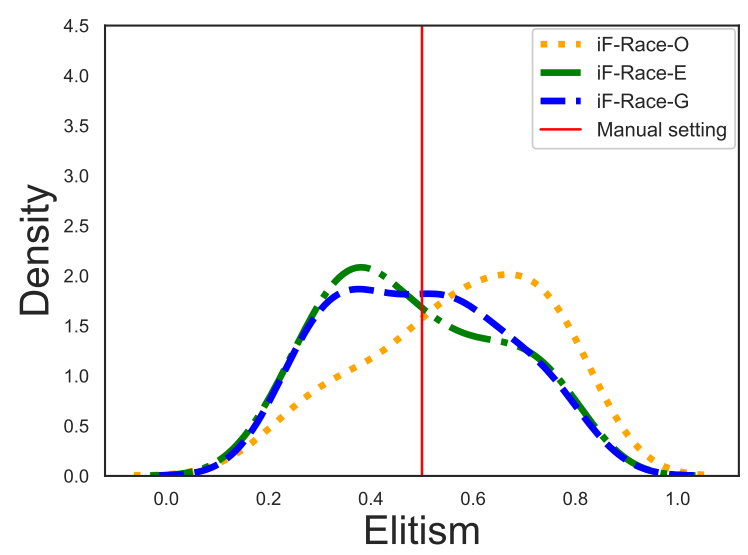

(d)

Fig. 1: Density plots for each hyper-parameter on the Koza \& Nguyen problems: (a) Species threshold; (b) Crossover Probability; (c) Species Criteria; and (d) Elitism. Each plot shows curves for the three iF-Race variants (Original, Elitism and Gaussian), and a vertical line for the manual configuration from (Trujillo et al, 2016).

performing parametrizations, these might not translate to substantial (if any) gains in testing error due to the possibility of overfitting.

A similar analysis for solution size is presented in Figure 7 and Figure 8 respectively for the Koza \& Nguyen and Pagie \& Korns problem sets. The plots show the average size of individuals in the final population of each GP search process, and they are very interesting. These results clearly show that the manual configuration evolves the smaller trees, and this is true for both sets of problems. In fact, this difference is much more pronounced on the more difficult problem set, Pagie \& Korns. On the one hand, results such as these seem very reasonable, since all of the iF-Race are exclusively focusing on predictive accuracy of the GP trees as measured by RMSE. The hyper-parameter optimization is not considering solution size, so there is no reason to expect that the optimized parametrizations should produce compact solutions or eliminate bloat. On the other hand, such a wide gap between the automatic and manual parametrizations was definitely unexpected. Moreover, the difference in solutions size is consistent across all problems, probably the most consistent result in all of our experiments.

\section{Discussion}

The most useful way to analyze the results presented thus far is to contrast them with the results presented by Sipper et al (2018). To do so, we will focus on some of the main findings by Sipper et al (2018), and discuss how our results can be viewed from that perspective. Before doing so, it is important to keep in mind three differences between both studies. First, Sipper et al (2018) considered common GP hyper-parameters, such 


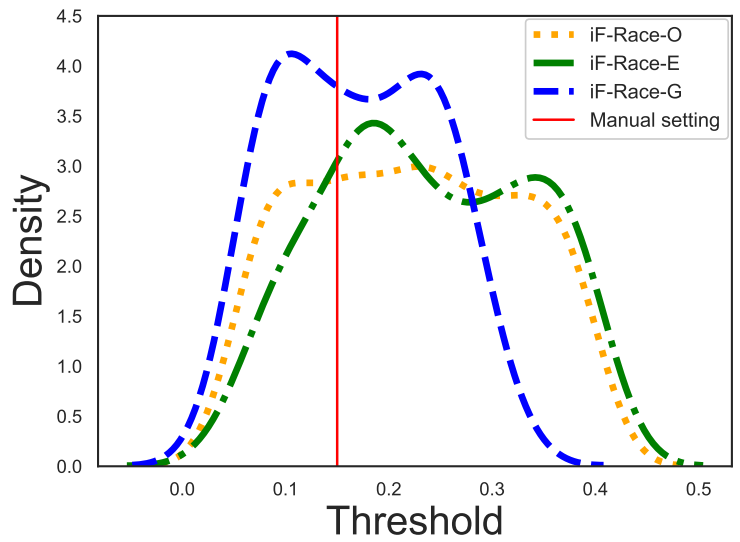

(a)

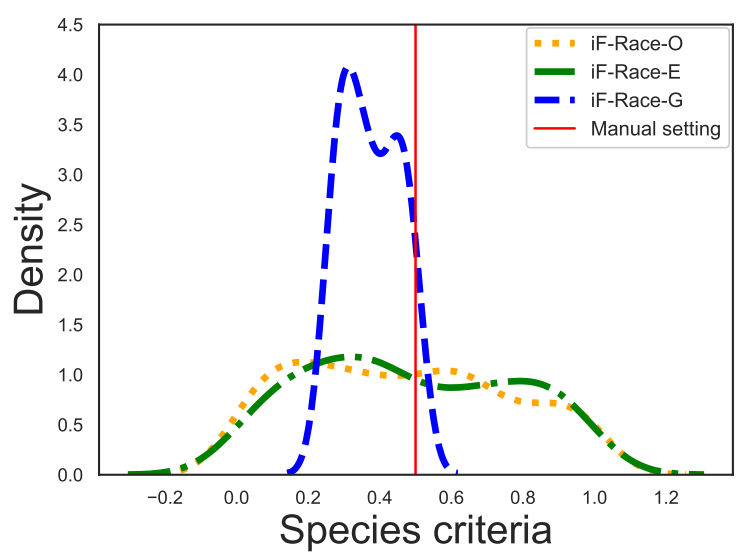

(c)

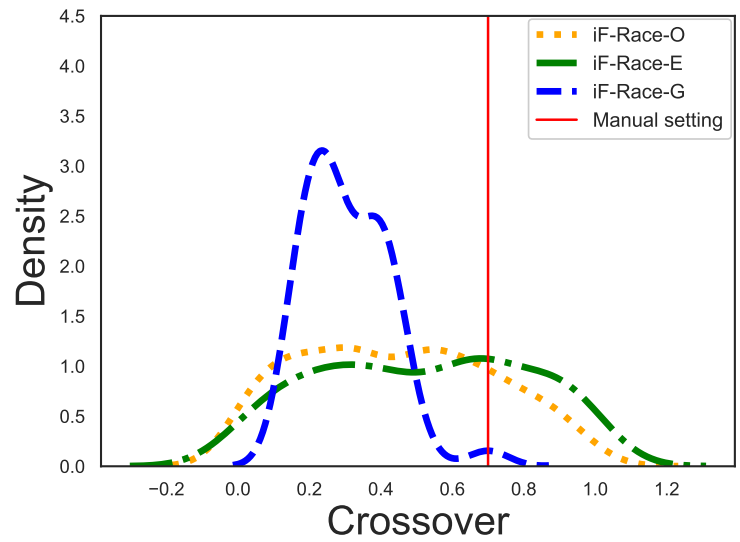

(b)

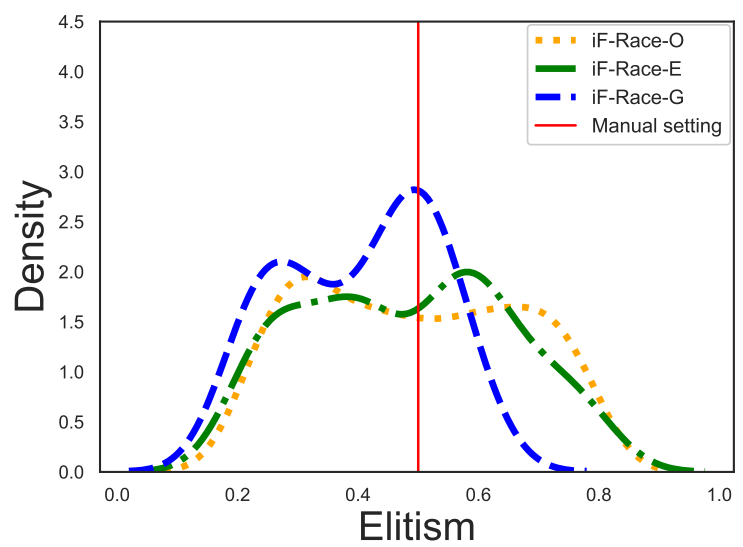

(d)

Fig. 2: Density plots for each hyper-parameter on the Pagie \& Korns problems: (a) Species threshold; (b) Crossover Probability; (c) Species Criteria; and (d) Elitism. Each plot shows curves for the three iF-Race variants (Original, Elitism and Gaussian), and a vertical line for the manual configuration from (Trujillo et al, 2016).

as population size, while the present work focused on more specialized parameters of the neat-GP algorithm. Second, Sipper et al (2018) used fitness (training performance) to evaluate the hyper-parameter optimization process, while the present work uses fitness to guide the hyper-parameter optimization process but also considers test performance and solution size in the subsequent analysis. Finally, Sipper et al (2018) used random search and an EA to perform hyper-parameter optimization, while this work uses iF-Race.

Sipper et al (2018) suggest that hyper-parameter space is rich with parametrizations that can achieve high levels of performance. In other words, that finding optimal values for single hyper-parameters is not a realistic goal, since there are strong dependencies between different parameters (for instance between population size and number of generations), such that many different values might lead to good performance when properly combined. The results presented in this paper, particular those summarized in figures 1 -4, seem to validate this assertion since at least two of the iF-Race variants (Original and Elitism) tend to cover almost the entire range of allowable parametrizations considered in our experiments.

Another conclusion from Sipper et al (2018) is that finding a single parametrization that works well for a single problem is relatively easy, but finding a parametrization that works well across multiple problems is a more difficult task. In general the results presented here cannot be said to contradict this conclusions, but do suggest that at the very least a better hyper-parametrization process (such as iF-Race) can consistently find parametrizations that actually generalize quite well across multiple problems.

On the other hand, some of the aspects not considered in the study by Sipper et al (2018), reveal the 


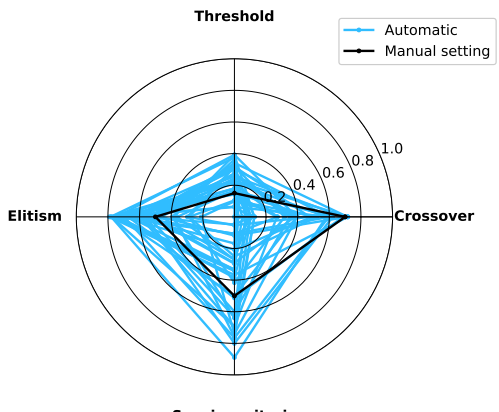

(a)

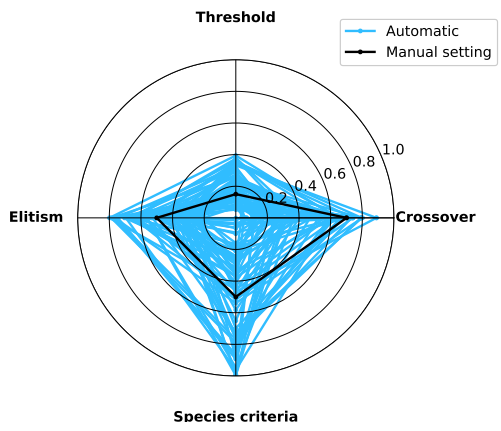

(b)

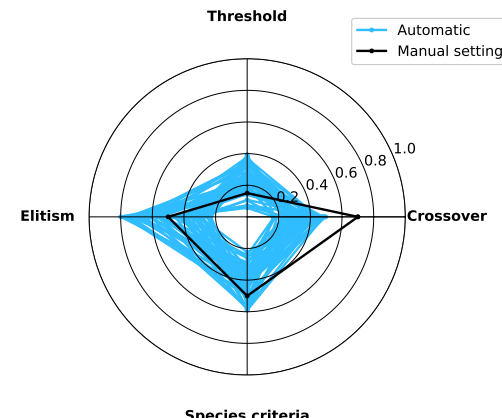

(c)

Fig. 3: Radar plots for each hyper-parameter on the Koza \& Nguyen problems: (a) Original; (b) Elitism; and (c) Gaussian. Each plot shows curves (darker line) for the manual configuration from (Truiillo et al, 2016).

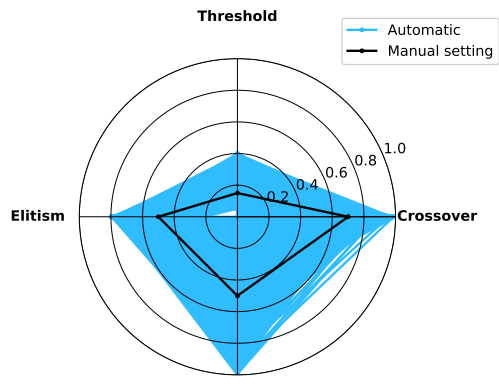

(a)

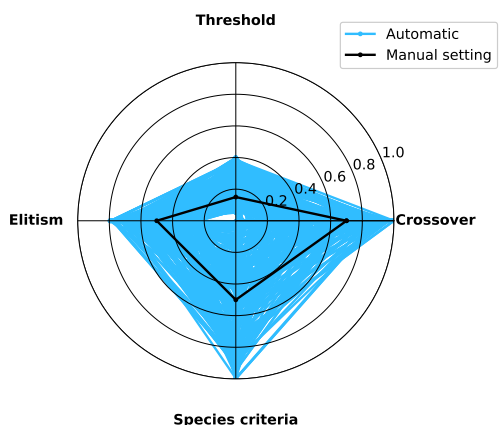

(b)

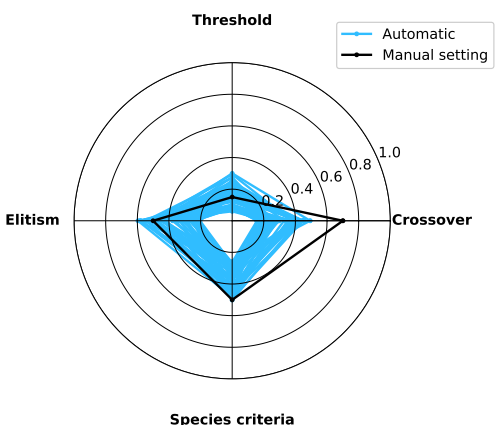

(c)

Fig. 4: Radar plots for each hyper-parameter on the Pagie \& Korns problems: (a) Original; (b) Elitism; and (c) Gaussian. Each plot shows curves (darker line) for the manual configuration from (Truillo et al, 2016).

following useful insights. First, that the manual configuration proposed in (Truililo et al, 2016) for neatGP is actually comparable to the elite parametrizations produced by iF-Race. While the search for optimal parametrizations seems to be difficult using automatic methods, an expert user can, at least in this case, find good configurations.

Second, the elite iF-Race parametrizations produce bloated GP runs. Notice that the manual parametrization relies on single parameter values that are similar to many of the elite configurations produced by iF-Race, clearly shown in the radar plots of figures 3 and 4 . One possible explanation might be to assume that the elite parametrizations produced by iF-Race overfit the problem data. However, this is not the case. Figure 9 and Figure ? show a comparisons of training RMSE between the elite parametrizations and the manual configuration. Clearly, the manual configuration shows lower training errors, this shows that the lack of bloat control by the elite parametrizations is not due to overfitting.

This suggests that the manual configuration is in fact unique, a just right setting that allows neat-GP to produce good solutions while also limiting the effect of bloat. If this is true, it could imply that the algorithm might be fragile, in the sense that even slight hyperparameter changes can have drastic effects on its ability to control bloat. Therefore, future work will focus on using a hyper-parameter optimization procedure that accounts for both performance measures (solution size and RMSE) concurrently.

\section{Concluding Remarks}

This paper presents an experimental characterization of the hyper-parameter space for neat-GP. This is work builds upon recent findings (Sipper et al, 2018), considering system specific parameters and considering other important aspects to consider in a GP system, namely test performance and size.

There are several conclusions to be drawn from this paper. GP systems, and particularly neat-GP, can produce good test error using a wide range of different hyper-parameter values. The iF-Race algorithm can consistently find parametrizations that compare favorably with a manual configuration in terms of testing RMSE. However, most of these parametrizations have 


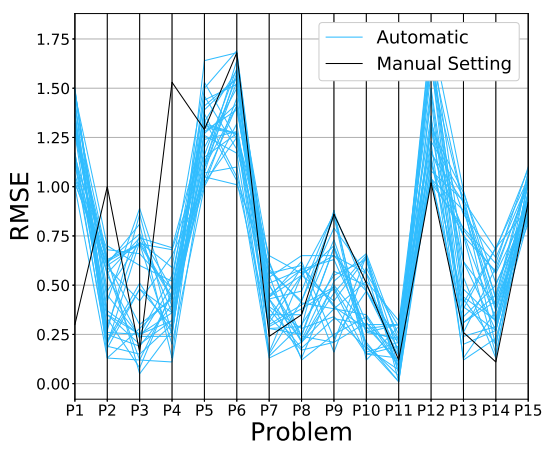

(a)

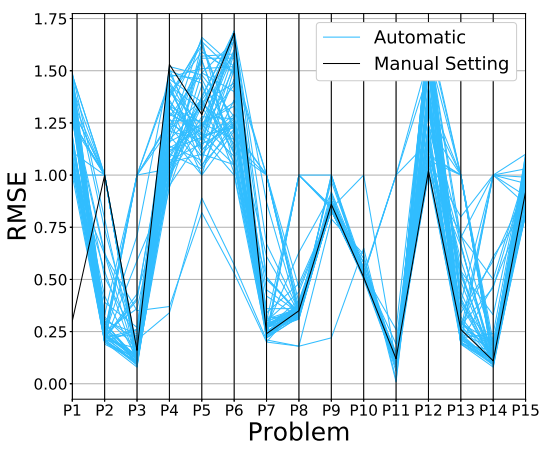

(b)

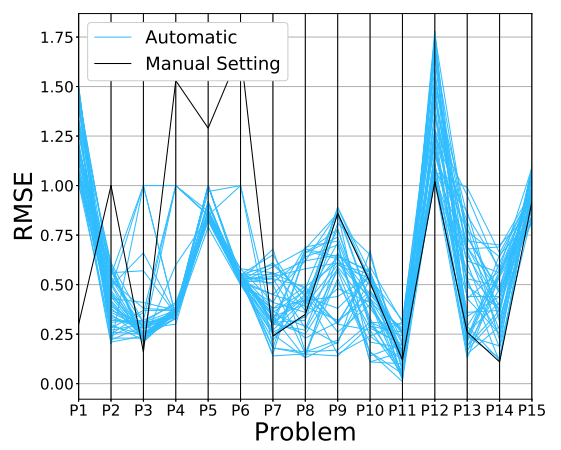

(c)

Fig. 5: Parallel coordinates plots for the test error (RMSE) on the Koza \& Nguyen problems: (a) Original; (b) Elitism; and (c) Gaussian. Each plot shows curves (darker line) for the manual configuration from (Trujillo et al, 2016).

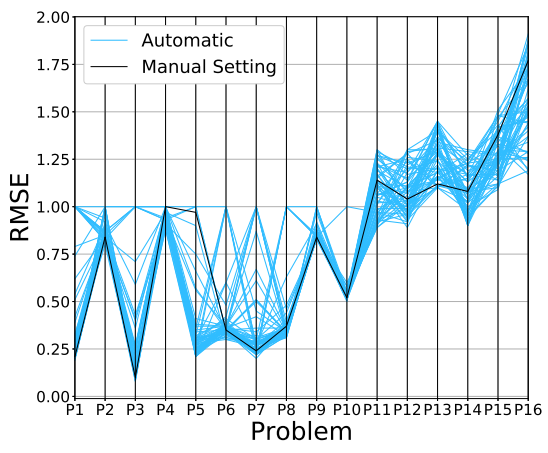

(a)

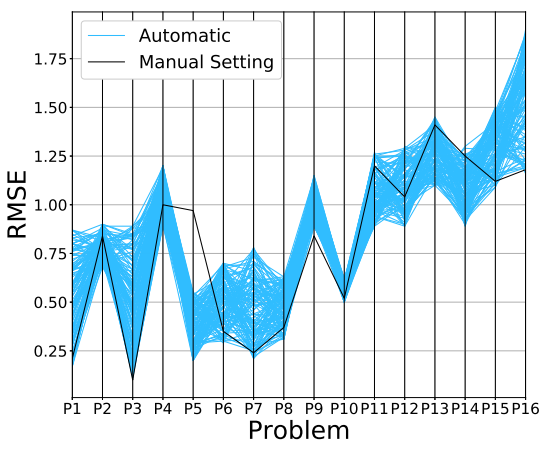

(b)

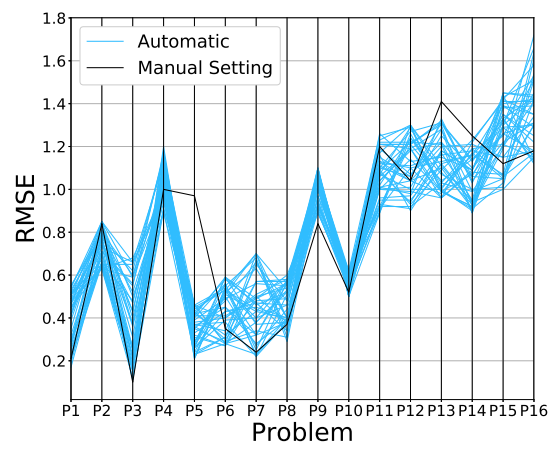

(c)

Fig. 6: Parallel coordinates plots for the test error (RMSE) on the Pagie \& Korns problems: (a) Original; (b) Elitism; and (c) Gaussian. Each plot shows curves (darker line) for the manual configuration from (Truiillo et al, 2016).

a negative effect on the ability of neat-GP to control bloat. This points to the need to perform multiobjective byper-parameter optimization, which would be a definite non-trivial tasks, and to the best of our knowledge has not been carried out in the GP community, opening up a great possibility for future work. The results shown also lead to the conclusion that the manual configuration reported in (Trujillo et al, 2016) is in fact fragile and unique. The manual configuration shows lower training errors and basically equal testing error compared to the automatic parametrizations. The individual parameter values do not seem unique, they are well within the ranges explored by iF-Race, but the specific combination used is clearly beneficial for bloat control.

From a more general perspective, this papers suggests that hyper-parameter optimization in GP is a difficult task to perform. This is true because GP can, and for many practitioners should, produce human readable models, the ability to do so is an advantage the method has over other learning methods. However, having to balance between both solution quality and solution size makes hyper-parameter optimization a more difficult computational task. Clearly showing the need for more research on this topic, which the reader is encouraged to explore.

\section{References}

Bergstra J, Bengio Y (2012) Random search for hyperparameter optimization. J Mach Learn Res 13:281305

Birattari M (2009) Tuning Metaheuristics: A Machine Learning Perspective, 1st edn. Springer Publishing Company, Incorporated

Birattari M, Stützle T, Paquete L, Varrentrapp K (2002) A racing algorithm for configuring metaheuristics. In: Proceedings of the 4th Annual Con- 


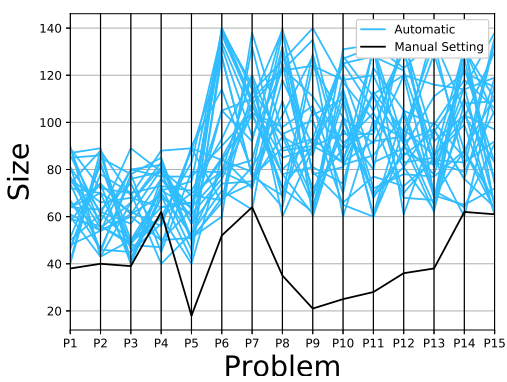

(a)

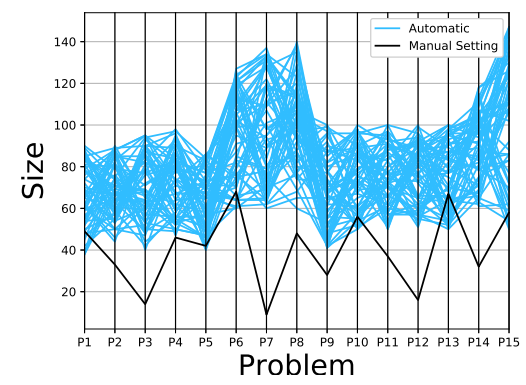

(b)

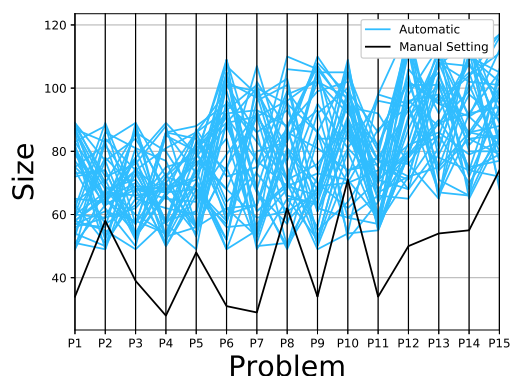

(c)

Fig. 7: Parallel coordinates plots for the average population size (number of nodes) in the last generation for the Koza \& Nguyen problems: (a) Original; (b) Elitism; and (c) Gaussian. Each plot shows curves (darker line) for the manual configuration from (Trujillo et al, 2016).

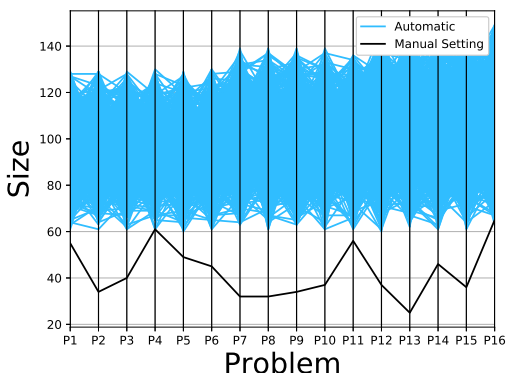

(a)

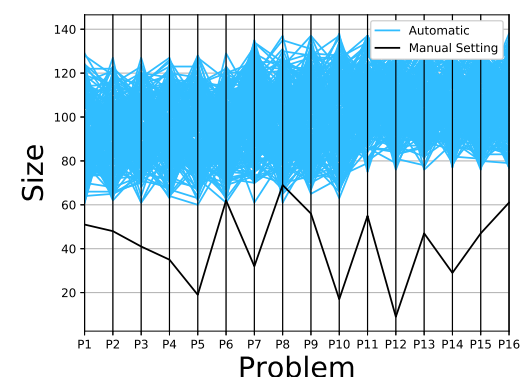

(b)

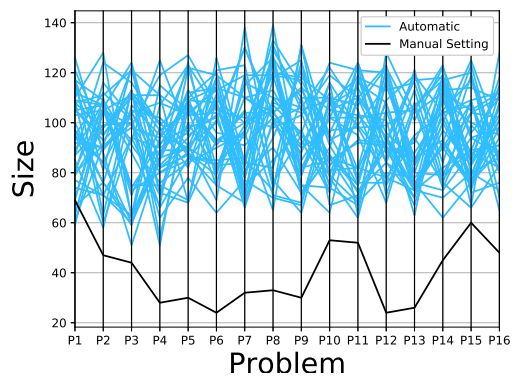

(c)

Fig. 8: Parallel coordinates plots for the average population size (number of nodes) in the last generation for the Pagie \& Korns problems: (a) Original; (b) Elitism; and (c) Gaussian. Each plot shows curves (darker line) for the manual configuration from (Trujillo et al, 2016).

ference on Genetic and Evolutionary Computation, Morgan Kaufmann Publishers Inc., San Francisco, CA, USA, GECCO'02, pp 11-18

Cava WL, Silva S, Danai K, Spector L, Vanneschi L, Moore JH (2019) Multidimensional genetic programming for multiclass classification. Swarm and Evolutionary Computation 44:260 - 272

De Rainville FM, Fortin FA, Gardner MA, Parizeau M, Gagné C (2012) Deap: A python framework for evolutionary algorithms. In: Proceedings of the 14th Annual Conference Companion on Genetic and Evolutionary Computation, ACM, New York, NY, USA, GECCO '12, pp 85-92

Hansen N, Ostermeier A (2001) Completely derandomized self-adaptation in evolution strategies. Evol Comput 9(2):159-195

Hernandez-Beltran JE, Diaz-Ramirez VH, Trujillo L, Legrand P (2019) Design of estimators for restoration of images degraded by haze using genetic programming. Swarm and Evolutionary Computation 44:4963
Juárez-Smith P, Trujillo L (2016) Integrating local search within neat-gp. In: Proceedings of the 2016 on Genetic and Evolutionary Computation Conference Companion, ACM, New York, NY, USA, GECCO '16 Companion, pp 993-996

Karafotias G, Hoogendoorn M, Eiben AE (2015) Parameter control in evolutionary algorithms: Trends and challenges. IEEE Transactions on Evolutionary Computation 19(2):167-187

Koza JR (1992) Genetic Programming: On the Programming of Computers by Means of Natural Selection. MIT Press, Cambridge, MA, USA

Langdon WB, Poli R (2010) Foundations of Genetic Programming, 1st edn. Springer Publishing Company, Incorporated

Lpez-Ibez M, Dubois-Lacoste J, Cceres LP, Birattari M, Sttzle T (2016) The irace package: Iterated racing for automatic algorithm configuration. Operations Research Perspectives 3:43- 58

McDermott J, White DR, Luke S, Manzoni L, Castelli M, Vanneschi L, Jaskowski W, Krawiec K, Harper R, 


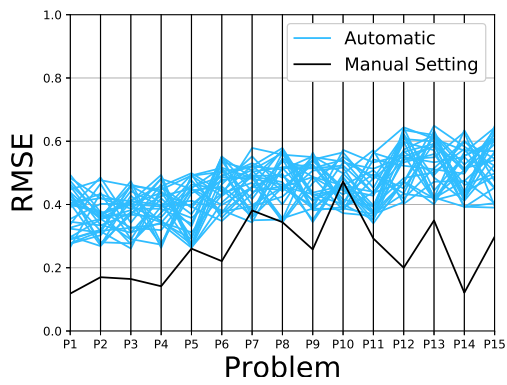

(a)

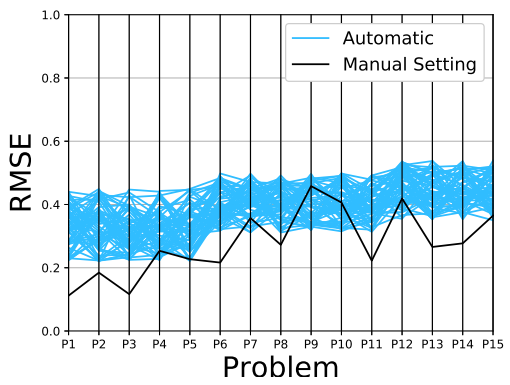

(b)

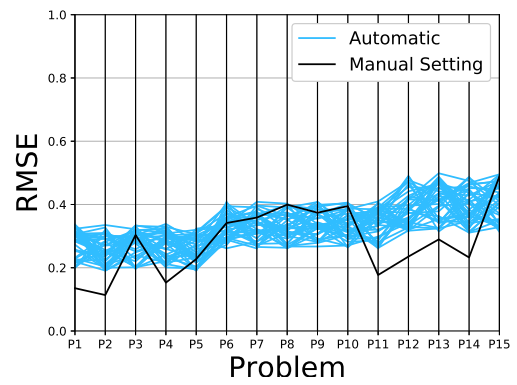

(c)

Fig. 9: Parallel coordinates plots for the training error (RMSE) on the Koza \& Nguyen problems: (a) Original; (b) Elitism; and (c) Gaussian. Each plot shows curves (darker line) for the manual configuration from (Truiillo et al, 2016).

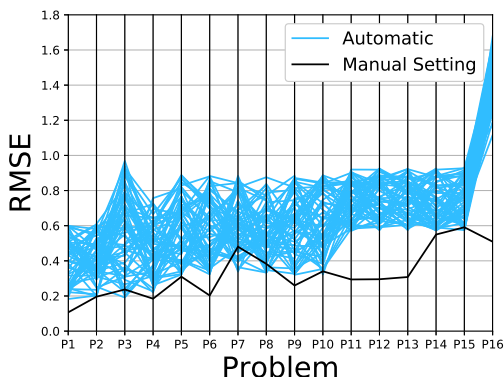

(a)

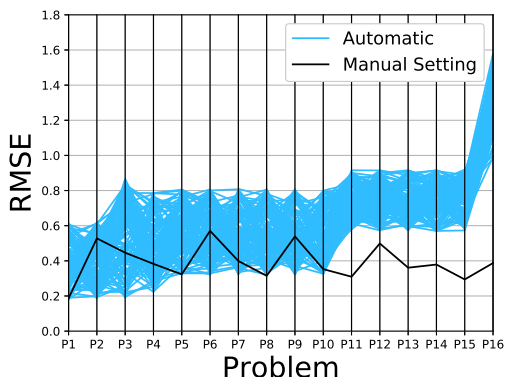

(b)

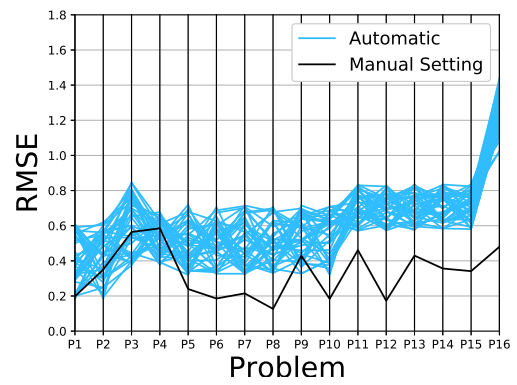

(c)

Fig. 10: Parallel coordinates plots for the training error (RMSE) on the Pagie \& Korns problems: (a) Original; (b) Elitism; and (c) Gaussian. Each plot shows curves (darker line) for the manual configuration from (Trujillo et al, 2016).

De Jong K, O'Reilly UM (2012) Genetic programming needs better benchmarks. In: Proceedings of the 14th Annual Conference on Genetic and Evolutionary Computation, ACM, New York, NY, USA, GECCO '12, pp 791-798

Neumüller C, Wagner S, Kronberger G, Affenzeller M (2012) Parameter meta-optimization of metaheuristic optimization algorithms. In: Proceedings of the 13th International Conference on Computer Aided Systems Theory - Volume Part I, Springer-Verlag, Berlin, Heidelberg, EUROCAST'11, pp 367-374

Olson RS, La Cava W, Orzechowski P, Urbanowicz RJ, Moore JH (2017) Pmlb: a large benchmark suite for machine learning evaluation and comparison. BioData Mining 10(1):36

Sipper M, Fu W, Ahuja K, Moore JH (2018) Investigating the parameter space of evolutionary algorithms. BioData Mining 11(1):2

Stanley KO, Miikkulainen R (2002) Evolving neural networks through augmenting topologies. Evol Comput 10(2):99-127
Trujillo L, Muoz L, Galvn-Lpez E, Silva S (2016) neat genetic programming: Controlling bloat naturally. Information Sciences 333:21 - 43 